\title{
DOUGLAS ALGEBRAS WITHOUT MAXIMAL SUBALGEBRA AND WITHOUT MINIMAL SUPERALGEBRA
}

CARROLL GUILLORY

Received 25 February 2001

We give several examples of Douglas algebras that do not have any maximal subalgebra. We find a condition on these algebras that guarantees that some do not have any minimal superalgebra. We also show that if $A$ is the only maximal subalgebra of a Douglas algebra $B$, then the algebra $A$ does not have any maximal subalgebra.

\section{Introduction}

Let $\mathbf{D}$ denote the open disk in the complex plane and $\mathbf{T}$ the unit circle. By $L^{\infty}$ we mean the space of essentially bounded measurable functions on $\mathbf{T}$ with respect to the normalized Lebesgue measure. We denote by $H^{\infty}$ the space of all bounded analytic functions in $\mathbf{D}$. Via identification with boundary functions, $H^{\infty}$ can be considered as a uniformly closed subalgebra of $L^{\infty}$. Any uniformly closed subalgebra $B$ strictly between $L^{\infty}$ and $H^{\infty}$ is called a Douglas algebra. $M(B)$ will denote the maximal ideal space of a Douglas algebra $B$. If we let $X=M\left(L^{\infty}\right)$, we can identify $L^{\infty}$ with $C(X)$, the algebra of continuous functions on $X$. If $C$ is the set of all continuous functions on $\mathbf{T}$, we set

$$
H^{\infty}+C=\left\{h+g: g \in C, h \in H^{\infty}\right\} .
$$

$H^{\infty}+C$ then becomes the smallest Douglas algebra containing $H^{\infty}$ properly. The function

$$
q(z)=\prod_{n=1}^{\infty} \frac{\left|z_{n}\right|}{z_{n}} \cdot \frac{z-z_{n}}{1-\bar{z}_{n} z}
$$

is called a Blaschke product if $\sum_{n=1}^{\infty}\left(1-\left|z_{n}\right|\right)$ converges. The set $\left\{z_{n}\right\}$ is called the zero set of $q$ in $\mathbf{D}$. Here $\left|z_{n}\right| / z_{n}=1$ is understood whenever $z_{n}=0$. We call 


\section{Douglas algebras}

$q$ an interpolating Blaschke product if

$$
\inf _{n} \prod_{m \neq n}\left|\frac{z_{m}-z_{n}}{1-\bar{z}_{n} z_{m}}\right|>0 .
$$

An interpolating Blaschke product $q$ is called sparse (or thin) if

$$
\lim _{n \rightarrow \infty} \prod_{m \neq n}\left|\frac{z_{m}-z_{n}}{1-\bar{z}_{n} z_{m}}\right|=1 .
$$

The set

$$
Z(q)=\left\{x \in M\left(H^{\infty}\right) \backslash \mathbf{D}: q(x)=0\right\}
$$

is called the zero set of $q$ in $M\left(H^{\infty}+C\right)$. Any function $h$ in $H^{\infty}$ with $|h|=1$, almost everywhere on $\mathbf{T}$, is called an inner function. Since $|q|=1$ for any Blaschke product, Blaschke products are inner functions. Let

$$
Q C=\left(H^{\infty}+C\right) \cap \overline{\left(H^{\infty}+C\right)}
$$

and, for $x \in M\left(H^{\infty}+C\right)$, set

$$
Q_{x}=\left\{y \in M\left(L^{\infty}\right): f(x)=f(y) \forall f \in Q C\right\} .
$$

$Q_{x}$ is called the $Q C$-level set for $x$. For $x \in M\left(H^{\infty}+C\right)$, we denote $u_{x}$ the representing measure for $x$ and its support set by supp $u_{x}$. By $H^{\infty}[\bar{q}]$ we mean the Douglas algebra generated by $H^{\infty}$ and the complex conjugate of the function $q$. Since $X$ is a Shilov boundary for every Douglas algebra, a closed set $E$ contained in $X$ is called a peak set for a Douglas algebra $B$ if there is a function in $B$ with $f=1$ on $E$ and $|f|<1$ on $X \backslash E$. A closed set $E$ is a weak peak set for $B$ if $E$ is the intersection of a family of peak sets. If the set $E$ is a weak peak set for $H^{\infty}$ and we define

$$
H_{E}^{\infty}=\left\{f \in L^{\infty}:\left.\left.f\right|_{E} \in H^{\infty}\right|_{E}\right\}
$$

then $H_{E}^{\infty}$ is a Douglas algebra. For a Douglas algebra $B, B_{E}$ is similarly defined. A closed set $E$ contained in $X$ is called the essential set for $B$, denoted ess $(B)$, if $E$ is the smallest set in $X$ with the property that for $f$ in $L^{\infty}$ with $f=0$ on $E$, then $f$ is in $B$.

For an interpolating Blaschke product $q$, we put $N(\bar{q})$ the closure of

$$
\bigcup\left\{\operatorname{supp} u_{x}: x \in M\left(H^{\infty}+C\right),|q(x)|<1\right\} .
$$

$N(\bar{q})$ is a weak peak set for $H^{\infty}$ and is referred to as the nonanalytic points of $q$. By $N_{0}(\bar{q})$ we denote the closure of

$$
\bigcup\left\{\operatorname{supp} u_{x}: x \in Z(q)\right\}
$$


For an $x \in M\left(H^{\infty}\right)$ we let

$$
E_{x}=\left\{y \in M\left(H^{\infty}\right): \operatorname{supp} u_{y}=\operatorname{supp} u_{x}\right\}
$$

and call $E_{x}$ the level set of $x$. Since the sets supp $u_{x}$ and $N(\bar{q})$ are weak peak sets for $H^{\infty}$, both $H_{\text {supp } u_{x}}^{\infty}$ and $H_{N(\bar{q})}^{\infty}$ are Douglas algebras. For any interpolating Blaschke product $q$ we set

$$
A=\bigcap_{x \in M\left(H^{\infty}+C\right) \backslash M\left(H^{\infty}[\bar{q}]\right)} H_{\operatorname{supp} u_{x}}^{\infty}, \quad A_{0}=\bigcap_{y \in Z(q)} H_{\operatorname{supp} u_{y}}^{\infty} .
$$

It is easy to see that $A \subseteq A_{0}$ and it was shown, in [7], that $A=H_{N(\bar{q})}^{\infty}$. For $x$ and $y$ in $M\left(H^{\infty}\right)$, the pseudo-hyperbolic distance is defined by

$$
\rho(x, y)=\sup \left\{|h(x)|:|h| \leq 1, h \in H^{\infty}, h(y)=0\right\} .
$$

For any $x \in M\left(H^{\infty}\right)$, we define the Gleason part of $x$ by

$$
P_{x}=\left\{y \in M\left(H^{\infty}\right): \rho(x, y)<1\right\} .
$$

If $P_{x} \neq\{x\}$, then $x$ is said to be a nontrivial point. We denote by $G$ the set of nontrivial points of $M\left(H^{\infty}+C\right)$, and for a Douglas algebra $B$, we set

$$
G_{B}=G \cap\left(M\left(H^{\infty}+C\right) \backslash M(B)\right) .
$$

A point $x$ in $G_{B}$ is called a minimal support point of $G_{B}$ (or simply a minimal support point of $B$ ) if there is no $y \in G_{B}$ such that supp $u_{y} \subseteq \operatorname{supp} u_{x}$. The set $\operatorname{supp} u_{x}$ is called a minimal support set for $B$. For Douglas algebras $B$ and $B_{0}$ with $B_{0} \subseteq B$ we let $\Omega\left(B, B_{0}\right)$ be all interpolating Blaschke products $q$ such that $\bar{q} \in B$ but $\bar{q} \notin B_{0}$.

We denote by $\Omega(B)$ the set of all interpolating Blaschke products $q$ with $\bar{q} \in B$. Let $B$ be a Douglas algebra. The Bourgain algebra $B_{b}$ of $B$ relative to $L^{\infty}$ is the set of those elements of $L^{\infty}, f$, such that $\left\|f f_{n}+B\right\|_{\infty} \rightarrow 0$ for every sequence $\left\{f_{n}\right\}$ in $B$ with $f_{n} \rightarrow 0$ weakly. The minimal envelop $B_{m}$ of a Douglas algebra $B$ is defined to be the smallest Douglas algebra which contains all minimal superalgebras of $B$. An algebra $A$ is called a minimal superalgebra of $B$ if, for all $x, y \in M(B) \backslash M(A), x \neq y$ implies supp $u_{x}=\operatorname{supp} u_{y}$.

\section{When two Douglas algebras have identical essential sets}

Consider the Douglas algebras $A$ and $A_{0}$ defined above. In [7], some conditions were given when $A \subseteq A_{0}$, but yet $\operatorname{ess}(A)=\operatorname{ess}\left(A_{0}\right)$. This happened because $\operatorname{ess}(A)=N(\bar{q})$ and $\operatorname{ess}\left(A_{0}\right)=N_{0}(\bar{q})$ (this is not hard to show). Theorem 1 of [7] gives conditions when $\operatorname{ess}(A) \neq \operatorname{ess}\left(A_{0}\right)$. The conditions found in [7, Theorem 5] are far more complicated than those found in Theorem 2.3 below. Yet ess $(A)=\operatorname{ess}\left(A_{0}\right)$ in that theorem [7, Theorem 5] and also satisfies the condition in Theorem 2.3. Before we state this theorem we need the following lemmas. 
Lemma 2.1. Let $A$ be any Douglas algebra and $q$ an interpolating Blaschke product with $\bar{q} \notin A$. Set $B=A[\bar{q}]$ and let $x \in M(A) \backslash M(B)$ whose support set is not trivial. Then $B_{\operatorname{supp} u_{x}}=A_{\operatorname{supp} u_{x}}[\bar{b}]$.

Proof. Since $A \subset B$, we have that $A_{\operatorname{supp} u_{x}} \subset B_{\operatorname{supp} u_{x}}$. Thus $M\left(B_{\operatorname{supp} u_{x}}\right) \subset$ $M\left(A_{\text {supp } u_{x}}\right)$. By the Chang Marshall theorem [1,11], it suffices to show that $M\left(B_{\operatorname{supp} u_{x}}\right)=M\left(A_{\operatorname{supp} u_{x}}[\bar{b}]\right)$. Let $y=M\left(B_{\operatorname{supp} u_{x}}\right)$. Then $y \in M\left(A_{\operatorname{supp} u_{x}}\right)$ and $|q(y)|=1$, since $M(B)=\{y \in M(A):|q(y)|=1\}$. Hence, $y \in M\left(A_{\text {supp } u_{x}}[\bar{q}]\right)$.

Now suppose $y \notin M\left(B_{\operatorname{supp} u_{x}}\right)$. If $y \notin M\left(A_{\operatorname{supp} u_{x}}\right)$, then $y \notin A_{\operatorname{supp} u_{x}}[\bar{b}]$ and we have nothing to prove. We assume that $y \in M\left(A_{\operatorname{supp} u_{x}}\right)$. Since $y \notin$ $M\left(B_{\text {supp } u_{x}}\right)$ implies that $|q(y)|<1$ and $y \in M\left(A_{\operatorname{supp} u_{x}}\right)$. Hence $y \notin$ $M\left(A_{\text {supp } u_{x}}[\bar{q}]\right)$. Thus $M\left(A_{\operatorname{supp} u_{x}}[\bar{q}]\right) \subset M\left(B_{\operatorname{supp} u_{x}}\right)$. We have that $M\left(A_{\operatorname{supp} u_{x}}[\bar{q}]\right)=M\left(B_{\operatorname{supp} u_{x}}\right)$. By the Chang-Marshall theorem, $A_{\operatorname{supp} u_{x}}[\bar{q}]=$ $B_{\text {Supp } u_{x}}$.

Lemma 2.2. Let $q$ be an interpolating Blaschke product and $x \in M\left(H^{\infty}+C\right)$ such that $|q(x)|<1$, and $\operatorname{supp} u_{x}$ is nontrivial. Put

$$
E=\cup\left\{\operatorname{supp} u_{y}: \operatorname{supp} u_{y} \subset \operatorname{supp} u_{x},|q(y)|+1\right\} .
$$

Then $E$ is a dense subset of $\operatorname{supp} u_{x}$.

Proof. To prove this, let $B_{1}=H_{\text {supp } u_{x}}^{\infty}$. Assume that $\bar{E}$, the closure of $E$, is properly contained in $\operatorname{supp} u_{x}$. Put

$$
B_{2}=\overline{H_{E}^{\infty}}=\overline{\left\{f \in L^{\infty}:\left.\left.f\right|_{E} \in H^{\infty}\right|_{E}\right\}}
$$

By [2, page 39], $M\left(B_{2}\right)=\left\{m \in M\left(H^{\infty}+C\right): \operatorname{supp} u_{m} \subseteq \bar{E}\right\} \cup M\left(L^{\infty}\right)$. Since $\bar{E} \subseteq \operatorname{supp} u_{x}$, we have that $B_{1} \subseteq B_{2}$. Therefore, $M\left(B_{2}\right) \subseteq M\left(B_{1}\right)$ and so there is a nontrivial point $x_{0} \in M\left(B_{1}\right) \backslash M\left(B_{2}\right)$ [4, Proposition 4.1] such that (a) $\operatorname{supp} u_{x_{0}} \subseteq \operatorname{supp} u_{x}$, (b) $\operatorname{supp} u_{x_{0}} \not \subset \bar{E}$ (otherwise $x_{0} \in M\left(B_{2}\right)$ ), (c) $\left|q\left(x_{0}\right)\right|<1$, and (d) $\operatorname{supp} u_{x_{0}} \cap E=\emptyset$.

By [6, Theorem 2], there is a $z_{0} \in Z(q)$ such that $\operatorname{supp} u_{z_{0}}$ is a minimal support set for $H^{\infty}[\bar{q}]$ that is contained in $\operatorname{supp} u_{x_{0}} \subseteq \operatorname{supp} u_{x}$. Since $q$ is an interpolating Blaschke product, $\operatorname{supp} u_{z_{0}}$ is not trivial. By [4, Theorem 4.2], there is an $m \in M\left(H^{\infty}+C\right)$ so that $\operatorname{supp} u_{m}$ is nontrivial and $\operatorname{supp} u_{m} \subseteq \operatorname{supp} u_{z_{0}} \subseteq$ $\operatorname{supp} u_{x_{0}}$. Since $\operatorname{supp} u_{x_{0}}$ is a minimal support set for $H^{\infty}[\bar{q}]$, we have that $|q(m)|=1$. Thus supp $u_{x_{0}} \cap E \neq \emptyset$. This contradicts (d). So $\bar{E}=\operatorname{supp} u_{x}$.

TheORem 2.3. Let $B_{0}$ be a subalgebra of a Douglas algebra $B$ with

$$
\operatorname{ess}\left(B_{0}\right) \neq \mathbf{X} \text {. }
$$

If for every $x \in M\left(B_{0}\right) \backslash M(B)$ we have $\operatorname{ess}\left(H_{\operatorname{supp} u_{x}}^{\infty}\right)=\operatorname{ess}\left(B_{\operatorname{supp} u_{x}}\right)$, then $\operatorname{ess}(B)=\operatorname{ess}\left(B_{0}\right)$. 
Proof. We note that ess $\left(H_{\operatorname{supp} u_{x}}^{\infty}\right)=\operatorname{supp} u_{x}$. Hence if ess $\left(H_{\operatorname{supp} u_{x}}^{\infty}\right)$ is contained in ess $(B)$ for every $x \in M\left(B_{0}\right) \backslash M(B)$, then $\operatorname{supp} u_{x} \subset \operatorname{ess}(B)$ for every $y \in$ $M\left(B_{0}\right)$ and so ess $\left(B_{0}\right) \subset \operatorname{ess}(B)$. Since $B_{0} \subset B$, we have that ess $(B) \subset \operatorname{ess}\left(B_{0}\right)$, and we get $\operatorname{ess}(B)=\operatorname{ess}\left(B_{0}\right)$.

Corollary 2.4. Let $B_{0}$ be a maximal subalgebra of a Douglas algebra $B$. Then $\operatorname{ess}\left(B_{0}\right)=\operatorname{ess}(B)$.

Proof. Since $M\left(B_{0}\right)=M(B) \cup E_{x}$ for some $x \in M\left(B_{0}\right) \backslash M(B)$, we have that if $z$ and $y$ are in $x \in M\left(B_{0}\right) \backslash M(B)$, then $\operatorname{supp} u_{y}=\operatorname{supp} u_{x}=\operatorname{supp} u_{z}$. Now $\operatorname{ess}\left(B_{\operatorname{supp} u_{x}}\right)=\operatorname{ess}\left(H_{\operatorname{supp} u_{x}}^{\infty}\right)$ since the set

$$
\bigcup\left\{\operatorname{supp} u_{y}: y \in M(B) \cap M\left(H_{\operatorname{supp} u_{x}}^{\infty}\right)\right\}
$$

is dense in $\operatorname{supp} u_{x}$ (because $x$ is a minimal point of $B$ ). Thus ess $(B)=\operatorname{ess}\left(B_{0}\right)$.

Corollary 2.5. Let $A$ be a Douglas algebra and $q$ an interpolating Blaschke product with $\bar{q} \notin A$. Then $\operatorname{ess}(A)=\operatorname{ess}(A[\bar{q}])$.

Proof. We have that $M(A)=\left\{x \in M\left(H^{\infty}+C\right): A_{\operatorname{supp} u_{x}}=H_{\operatorname{supp} u_{x}}^{\infty}\right\}$. By Lemma 2.1, we have

$$
\operatorname{ess}\left(A[\bar{q}]_{\operatorname{supp} u_{x}}\right)=\operatorname{ess}\left(A_{\operatorname{supp} u_{x}}[\bar{q}]\right)=\operatorname{ess}\left(H_{\operatorname{supp} u_{x}}^{\infty}\right)
$$

But by Lemma 2.2, we have that

$$
\operatorname{ess}\left(H_{\operatorname{supp} u_{x}}^{\infty}[\bar{q}]\right)=\operatorname{ess}\left(H_{\operatorname{supp} u_{x}}^{\infty}\right)
$$

Hence

$$
\operatorname{ess}\left(A[\bar{q}]_{\operatorname{supp} u_{x}}\right)=\operatorname{ess}\left(H_{\operatorname{supp} u_{x}}^{\infty}\right)=\operatorname{supp} u_{x}
$$

for all $x \in M(A) \backslash M(A[\bar{q}])$. By Theorem 2.3 the corollary follows.

We mention here that Corollary 2.5 was proved in [13, Theorem 2] by another method.

There are algebras $B_{0}$ and $B$ that satisfy the hypothesis of Theorem 2.3 and are not of the form $B=B_{0}[\bar{q}]$ for any interpolating Blaschke product (if $\left.B_{0} \subseteq B\right)$. To see this, let $\Gamma$ be the collection of sparse Blaschke products and $B$ the Douglas algebra $\left[H^{\infty}: \bar{q} ; q \in \Gamma\right]$. Let $q_{0}$ be an element in $\Gamma$ and put $B_{0}=H^{\infty}[\bar{q}]$. Then $B_{0} \subset B$. By a theorem of Hedenmaln [9], we have that if $b$ is a Blaschke product such that $\bar{b} \in B$, then $b=b_{1} \cdots b_{n}$, where each $b_{i}$, $i=1, \ldots, n$, is a sparse Blaschke product. Hence if $x \in M\left(B_{0}\right) \backslash M(B)$, then $x$ is the zero of some sparse Blaschke product. So ess $\left(B_{\operatorname{supp} u_{x}}\right)=\operatorname{ess}\left(H_{\operatorname{supp} u_{x}}^{\infty}\right)$. 
So, by Theorem 2.3, we have ess $(B)=\operatorname{ess}\left(B_{0}\right)$. (Theorem 3.1 below shows that $H_{\mathrm{supp} u_{x}}^{\infty}$ is a maximal subalgebra of $B_{\operatorname{supp} u_{x}}$.) Now suppose there is a Blaschke product $q \in \Omega\left(B, B_{0}\right)$ with $B=B_{0}[\bar{q}]$. Again by Hedenmaln's theorem, we have $q=q_{1} \cdots q_{n}$ with each $q_{i}$ a sparse Blaschke product. Let $Q$ be an infinite sparse Blaschke product such that $|Q|=1$ on $\cup_{x \in Z(q)} P_{x}$. Then, there is an $m \in M\left(H^{\infty}+C\right)$ such that $Q(m)=0$ but $m \notin \cup_{x \in Z(q)} P_{x}$. Thus $|q(m)|=1$ and so we get that $m \in M\left(B_{0}[\bar{q}]\right)$. Thus $\bar{Q} \notin B_{0}[\bar{q}]$ and yet $\bar{Q} \in B$. This implies that $B_{0}[\bar{q}] \subseteq B$, which is a contradiction

\section{Maximal subalgebras that have no maximal subalgebras}

We begin by extending [5, Proposition 1]. There, the authors showed that if $x \in Z(q)$ with $q$ a sparse Blaschke product, then the algebra $H_{\operatorname{supp} u_{x}}^{\infty}$ is a maximal subalgebra of $H_{\mathrm{supp} u_{x}}^{\infty}[\bar{q}]$. Below we show that this is true for a larger class.

Theorem 3.1. Let $A$ be any Douglas algebra with maximal subalgebra and $x a$ minimal support point of $G_{A}$. Then $H_{\operatorname{supp} u_{x}}^{\infty}$ is a maximal subalgebra of $A_{\operatorname{supp} u_{x}}$ and $A_{\operatorname{supp} u_{x}}=H_{\operatorname{supp} u_{x}}^{\infty}[\bar{q}]$ for some $q \in \Omega(A)$.

Proof. Let $B_{0}=H_{\text {supp } u_{x}}^{\infty}$ and $B=A_{\text {supp } u_{x}}$. Suppose $x$ is a minimal support set for $G_{A}$. Then we have, for any interpolating Blaschke product $\psi \in \Omega(A)$ with $|\psi(x)|<1$ and any $y \in M\left(H^{\infty}+C\right)$ with supp $u_{y} \subseteq \operatorname{supp} u_{x},|\psi(y)|=1$. Thus if $\psi_{0} \in \Omega(B)$ and $\left|\psi_{0}(x)\right|<1$, there is a $\psi \in \Omega(A)$ such that $\left.\psi\right|_{\operatorname{supp} u_{x}}=$ $\left.\psi_{0}\right|_{\text {supp } u_{x}}$. This implies that $\left|\psi_{0}(y)\right|=1$ for every such $y$. Hence $x$ is a minimal support point for $G_{B}$. Note that this implies that $M(B)=M\left(B_{0}\right) \backslash E_{x}$, where each $E_{x}$ is a level set for $x$. Hence $M\left(B_{0}\right)=M(B) \cup E_{x}$, so by [6, Theorem 1], $B_{0}$ is a maximal subalgebra of $B$. Let $q$ be any element in $\Omega(A)$ with $q(x)=0$. Then $q \in \Omega\left(B, B_{0}\right)$, and we have that $B=B_{0}[\bar{q}]$.

We will need the following lemmas in the proof of Theorem 3.4 below.

Lemma 3.2. For distinct points $x_{1}$ and $x_{2}$ in $G_{A}$, there is an interpolating Blaschke product $b$ such that $\bar{b} \in A$ and $b\left(x_{1}\right)=b\left(x_{2}\right)=0$.

Proof. Let $b_{1}$ and $b_{2}$ be interpolating Blaschke products with $b_{1}\left(x_{1}\right)=b_{2}\left(x_{2}\right)=$ 0 . Take two open subsets $V_{1}$ and $V_{2}$ of $M\left(H^{\infty}\right)$ such that $x_{i} \in \bar{V}_{i}, V_{i} \cap M(A)=$ $\emptyset$, and $\bar{V}_{1} \cap \bar{V}_{2}=\emptyset$, where $\bar{V}_{i}$ is the closure of $V_{i}$ in $M\left(H^{\infty}\right), i=1,2$. Let $\psi_{i}$ be a subproduct of $b_{i}$ whose zeros are zeros of $b_{i}$ in $V_{i}$. Then it is not hard to see that $b=\psi_{1} \psi_{2}$ is the desired function.

To prove Lemma 3.3, we assume that $\operatorname{supp} u_{x}$ is not a one point set for every $x \in G_{A}$. 
LeMmA 3.3. Let $x$ and $y$ be distinct points in $G_{A}$ with $\operatorname{supp} u_{y} \not \subset \operatorname{supp} u_{x}$. Then there is an interpolating Blaschke product $b$ such that $b \in A,|b(x)|=1$, and $b(y)=0$.

Proof. By Lemma 3.2, there is an interpolating Blaschke product $\psi$ with zeros $\left\{z_{n}\right\}_{n=1}^{\infty}$ such that $\bar{\psi} \in A$ and $\psi(x)=\psi(y)=0$. Since supp $u_{y} \not \subset \operatorname{supp} u_{x}$, there is an open and closed subset $U$ of $M\left(L^{\infty}\right)$ such that supp $u_{x} \subset U$, supp $u_{y} \not \subset U$, and supp $u_{y} \not \subset M\left(L^{\infty}\right) \backslash U$. For the characteristic function $\chi_{U}$ on $M\left(L^{\infty}\right)$, put $\hat{\chi}_{U}(\lambda)=\int_{X} \chi_{U} d u_{\lambda}$ for every $\lambda \in M\left(H^{\infty}\right)$. Then $\hat{\chi}_{U}$ is a continuous function on $M\left(H^{\infty}\right), \hat{\chi}_{U}(y)<1$ [9, page 93]. Let

$$
\left\{w_{n}\right\}_{n=1}^{\infty}=\left\{z_{p}: \hat{\chi}_{U}\left(z_{p}\right)<\frac{1+\hat{\chi}_{U}(y)}{2}\right\}
$$

and let $b$ be an interpolating Blaschke product with zeros $\left\{w_{n}\right\}_{n=1}^{\infty}$. Then $\bar{b} \in A$. Since $z(\psi)$ coincides with the closure of $\left\{z_{p}\right\}_{p=1}^{\infty}$ in $M\left(H^{\infty}\right)$ [10, page 205], $y$ is contained in the closure of $\left\{w_{n}\right\}_{n=1}^{\infty}$. Hence $b(y)=0$. To prove $|b(x)|=1$, suppose $|b(x)|<1$ and $b(m)=0$. Then we have $\hat{\chi}_{U}(m)=1$. Since $b(m)=0$, $m$ is contained in the closure of $\left\{w_{n}\right\}_{n=1}^{\infty}$, so that $\hat{\chi}_{U}(m) \leq\left(1+\hat{\chi}_{U}(y) / 2\right)<1$. This is a contradiction. So $|b(x)|=1$. The lemma follows.

Theorem 3.4. A Douglas algebra A has no maximal subalgebra if and only if $H_{\mathrm{supp} u_{x}}^{\infty}$ is not a maximal subalgebra of $A_{\operatorname{supp} u_{x}}$ for every $x \in G_{A}$.

Proof. Suppose $A$ has no maximal subalgebra and let $x \in G_{A}$. Since $x$ is not a minimal support point of $G_{A}$, there is a $y \in G_{A}$ with $\operatorname{supp} u_{y} \subseteq \operatorname{supp} u_{x}$, and a $\psi \in \Omega$ such that $|\psi(y)|<1$. Since $\bar{\psi} \notin H_{\text {supp } u_{y}}^{\infty}$, we can assume that $\psi(y)=0$. Hence $y \notin M\left(A_{\operatorname{supp} u_{x}}\right)$. By Lemma 3.3 , there is a $\psi_{0} \in \Omega\left(A_{\operatorname{supp} u_{x}}\right)$ such that $\left|\psi_{0}(y)\right|=1$ and $\psi_{0}(x)=0$. Then we have $H_{\text {supp } u_{x}}^{\infty} \subseteq H_{\text {supp } u_{x}}^{\infty}\left[\bar{\psi}_{0}\right] \subseteq A_{\operatorname{supp} u_{x}}$. So $H_{\operatorname{supp} u_{x}}^{\infty}$ is not a maximal subalgebra of $A_{\operatorname{supp} u_{x}}$.

Suppose that for all $x \in G_{A}, H_{\operatorname{supp} u_{x}}^{\infty}$ is not a maximal subalgebra of $A_{\operatorname{supp} u_{x}}$. Then there is an algebra $B$ with $H_{\operatorname{supp} u_{x}}^{\infty} \subseteq B \subseteq A_{\text {supp } u_{x}}$. Thus we can find a $y \in M\left(H^{\infty}+C\right)$ such that $\operatorname{supp} u_{y} \subseteq \operatorname{supp} u_{x}$ and $y \in M(B) \backslash M\left(A_{\operatorname{supp} u_{x}}\right)$. This implies that there is an interpolating Blaschke product $q$ with $\bar{q} \in B \subset$ $A_{\text {supp } u_{x}}$ such that $|q(y)|=1$ and $|q(x)|<1$. Hence there is a $q_{0} \in \Omega(A)$ with $\left.q_{0}\right|_{\operatorname{supp} u_{x}}=\left.q\right|_{\operatorname{supp} u_{x}}$. So $\left|q_{0}(y)\right|=1$ and $\left|q_{0}(x)\right|<1$. This implies that $x$ is not a minimal support point of $G_{A}$ for every $x \in G_{A}$. So by [6, Theorem 1], $A$ has no maximal subalgebra.

Proposition 3.5. Let $x \in M\left(H^{\infty}\right) \backslash M\left(L^{\infty}\right)$. Then $H_{\mathrm{supp} u_{x}}^{\infty}$ has no maximal subalgebra.

Proof. Now ess $\left(H_{\operatorname{supp} u_{x}}^{\infty}\right)=\operatorname{supp} u_{x}$. Hence if $y \in G_{H_{\text {supp } u_{x}}^{\infty}}$, then supp $u_{y} \cap$ $\operatorname{supp} u_{x}=\emptyset$. Hence if $A$ is a subalgebra of $H_{\operatorname{supp} u_{x}}^{\infty}$, there is a $y \in$ 
$M(A) \backslash M\left(H_{\text {supp } u_{x}}^{\infty}\right)$ with $\operatorname{supp} u_{y} \cap \operatorname{supp} u_{x}=\emptyset$ or $\operatorname{supp} u_{x} \subset \operatorname{supp} u_{y}$. Hence $\operatorname{ess}(A) \supseteq \operatorname{supp} u_{y} \cup \operatorname{supp} u_{x} \supseteq \operatorname{supp} u_{x}=\operatorname{ess}\left(H_{\operatorname{supp} u_{x}}^{\infty}\right)$. By Corollary $2.4, A$ is not a maximal subalgebra of $H_{\operatorname{supp} u_{x}}^{\infty}$.

Proposition 3.6. Let A be a Douglas algebra that has only one maximal subalgebra $A_{0}$. Then $A_{0}$ has no maximal subalgebra.

Proof. Suppose there is a subalgebra $B_{0} \subseteq A_{0}$ such that $B_{0}$ is a maximal subalgebra of $A_{0}$. Then, by [5, Theorem 1], there is an $x_{0} \in G_{A_{0}}$ such that

$$
M\left(B_{0}\right)=M\left(A_{0}\right) \cup E_{x_{0}} .
$$

Since $A_{0}$ is a maximal subalgebra of $A$, there is an $x \in G_{A} \cap M\left(B_{0}\right)$ such that

$$
M\left(A_{0}\right)=M(A) \cup E_{x} .
$$

By (3.2) and (3.3), we have that $M\left(B_{0}\right)=M(A) \cup E_{x} \cup E_{x_{0}}$. Since $x \in M\left(A_{0}\right)$, we have that $\operatorname{supp} u_{x} \neq \operatorname{supp} u_{x_{0}}$. Also since $x_{0} \notin E_{x}$ and $x_{0} \in G_{A_{0}}$, we have that $\operatorname{supp} u_{x_{0}} \not \subset \operatorname{supp} u_{x}$ (otherwise $x_{0} \in M(A)$ by (3.3)). We show that $x_{0}$ is a minimal support point of $G_{A}$, and hence get a contradiction. Let $y \in M\left(H^{\infty}+\right.$ $C)$ such that $y \in M\left(A_{0}\right)=M(A) \cup E_{x}$. If $y \in M(A)$, then we are done. So we can assume that $y \in E_{x}$. If $y \in E_{x}$, then $\operatorname{supp} u_{y}=\operatorname{supp} u_{x}$, so we have that $\operatorname{supp} u_{x} \subseteq \operatorname{supp} u_{x_{0}}$. Since $x \notin M(A)$, there is an interpolating Blaschke product $q$ with $\bar{q} \in A$ and such that $q(x)=0$. By [7, Theorem 2], there is an uncountable set $U$ of $Z(q)$ such that (a) $\operatorname{supp} u_{m} \subseteq \operatorname{supp} u_{x_{0}}$ for all $m \in U$ and (b) $\operatorname{supp} u_{m} \cap \operatorname{supp} u_{k}$ for all $m, k \in U, m \neq k$. By (3.2), each such $m \in U$ is in $M\left(A_{0}\right)$. Since for all $m \in U$ (except if $m=x$ ) we have $\operatorname{supp} u_{x} \cap \operatorname{supp} u_{m}=$ $\emptyset$, hence by (3.3), $m \in M(A)$. But $\bar{q} \in A$ and $U \subset Z(q) \cap M(A)$. This is a contradiction, and we get $y \notin E_{x}$. So $y \in M(A)$ and since $\operatorname{supp} u_{x_{0}} \neq \operatorname{supp} u_{x}$, we have that $x_{0}$ is a minimal support point of $G_{A}$. This is a contradiction. So $A_{0}$ has no maximal subalgebra.

Note that Proposition 3.5 follows from Proposition 3.6 if $x$ is a minimal support point for some interpolating Blaschke product.

Let $q$ be an interpolating Blaschke product. We consider the algebra $H_{N(\bar{q})}^{\infty}$. Certainly $H_{N(\bar{q})}^{\infty}$ is not known to be a maximal subalgebra of any Douglas algebra, but it does have some of the same properties of $H_{\operatorname{supp} u_{x}}^{\infty}$. For example, we have the following proposition.

Proposition 3.7. The algebra $H_{N(\bar{q})}^{\infty}$ has no maximal subalgebra.

Proof. Set $B=H_{N(\bar{q})}^{\infty}$. Let $x \in G_{B}$ and suppose $x$ is a minimal support point for $G_{B}$. Then if $y \in M\left(H^{\infty}+C\right)$ such that $\operatorname{supp} u_{y} \subseteq \operatorname{supp} u_{x}$, we have that $y \in M(B)$. By [2, page 39], we must have that $\operatorname{supp} u_{y} \subseteq N(\bar{q})=\operatorname{ess}(B)$. Thus 
we have that $\operatorname{ess}(B) \cap \operatorname{supp} u_{x}=N(\bar{q}) \cap \operatorname{supp} u_{x} \neq \emptyset$. By [10, Theorem 1], $N(\bar{q})=\cup_{x \in Z(q)} Q_{x}$. So there is an $x_{0} \in Z(q)$ such that $\operatorname{supp} u_{x} \cap Q_{x_{0}} \neq \emptyset$. By the definition of $Q_{x_{0}}$, we have that supp $u_{x} \subset Q_{x_{0}}$. By [2, page 39], this implies that $x \in M(B)$, which is a contradiction. So if $x \in G_{B}$, then supp $u_{x} \cap N(\bar{q})=\emptyset$, which implies that $x$ is not a minimal support point for $G_{B} . B$ has no maximal subalgebra.

\section{Minimal superalgebras of $H_{\operatorname{supp} u_{x}}^{\infty}$}

We will compute the Bourgain algebras and the minimal envelops of the Douglas algebra $H_{\operatorname{supp} u_{x}}^{\infty}$ for any $x \in M\left(H^{\infty}+C\right)$. We have the following theorem.

TheOREM 4.1. Let $x \in M\left(H^{\infty}+C\right) \backslash M\left(L^{\infty}\right)$ such that $|q(x)|<1$ for some interpolating Blaschke product $q$, and set $B=H_{\operatorname{supp} u_{x}}^{\infty}$. Then

(i) Either $B_{b}=B$ or $B_{b}=B[\bar{\psi}]$ for some interpolating Blaschke product $\psi$.

(ii) Either $B_{m}=B_{b}=B$ or $B_{m}=B[\bar{\psi}]$ for some interpolating Blaschke product $\psi$.

Proof. We will use [3, Theorem 2] which says that for any interpolating Blaschke product $\psi$ with $\bar{\psi} \in B_{b}$, we have $Z(\psi) \cap M(B)$ is a finite set and the fact that

$$
M(B)=M\left(L^{\infty}\right) \cup\left\{m \in M\left(H^{\infty}\right): \operatorname{supp} u_{m} \subseteq \operatorname{supp} u_{x}\right\} .
$$

We claim that if $\psi$ is an interpolating Blaschke product such that $\bar{\psi} \in B_{b}$, then $Z(\psi) \cap M(B) \subset E_{x}$, the level set of $x$. Suppose not, then there is an $x_{0} \in Z(\psi) \cap M(B)$ such that $\operatorname{supp} u_{x_{0}} \subseteq \operatorname{supp} u_{x}$. By [7, Theorem 2], there is an uncountable set $\Gamma$ of $Z(\psi)$ such that (a) $\operatorname{supp} u_{\gamma} \subseteq \operatorname{supp} u_{x}$ for all $\gamma \in \Gamma$ and (b) $\operatorname{supp} u_{m} \cap \operatorname{supp} u_{\gamma}=\emptyset$ for all $m, \gamma \in \Gamma, m \neq \gamma$. By (a) and (4.1), each $\gamma \in M(B)$ and so $\Gamma \subset Z(\psi) \cap M(B)$. This implies that the set $Z(\psi) \cap M(B)$ is infinite. This is a contradiction. Hence if $x_{0} \in Z(\psi) \cap M(B)$, then supp $u_{x_{0}}=$ supp $u_{x}$, so we get $Z(\psi) \cap M(B) \subset E_{x}$. There are two possibilities. (1) The set $Z(\psi) \cap M(B)=\emptyset$ for which $\psi \in B$, so $B_{b} \subseteq B$. This gives the case when $B_{b}=B$.

(2) If $Z(\psi) \cap M(B) \neq \varnothing$ but is finite. Then the algebra $B[\bar{\psi}] \subseteq B_{b}$. To show that $B_{b}=B[\bar{\psi}]$, let $\psi_{0}$ be another interpolating Blaschke product with $\bar{\psi}_{0} \in B_{b}$. Since both sets $Z\left(\psi_{0}\right) \cap M(B)$ and $Z(\psi) \cap M(B)$ are contained in $E_{x}$, we have that $M(B) \backslash M\left(B\left[\bar{\psi}_{0}\right]\right)=M(B) \backslash M(B[\bar{\psi}])=E_{x}$. Thus $M\left(B\left[\bar{\psi}_{0}\right]\right)=$ $M(B[\bar{\psi}])$, and by the Chang-Marshall theorem $[1,11]$ we have $B\left[\bar{\psi}_{0}\right]=B[\bar{\psi}]$. Since this is true for all $\psi, \psi_{0}$, we have by [8, Theorem C], $B_{b}=B[\psi]$ for any such $\psi(B[\bar{\psi}]$ is a minimal superalgebra of $B)$. This proves (i).

To prove (ii) let $\bar{\psi} \in B_{m}$. Then by [8, Theorem 3], there is a finite set $\left\{x_{1}, \ldots, x_{n}\right\} \subset Z(\psi) \cap M(B)$ such that $\{u \in M(B):|\psi(u)|<1\}=E_{x_{1}} \cup \cdots \cup$ $E_{x_{n}}$. Again we claim that $E_{x_{1}}=E_{x_{2}}=\cdots=E_{x_{n}}=E_{x}$. Suppose that $E_{x_{1}} \neq$ 
$E_{x_{2}}$. Then $\operatorname{supp} u_{x_{1}} \neq \operatorname{supp} u_{x_{2}}$. By (4.1) either $\operatorname{supp} u_{x_{1}} \subseteq \operatorname{supp} u_{x}$ or $\operatorname{supp} u_{x_{2}} \subseteq$ $\operatorname{supp} u_{x}$ or both. Suppose that $\operatorname{supp} u_{x_{1}} \subseteq \operatorname{supp} u_{x}$. Then by [7, Theorem 2], there is an uncountable set $\Gamma$ such that $E_{\alpha} \neq E_{\beta}$ for all $\alpha, \beta \in \Gamma$ and $\cup_{\alpha \in \Gamma} E_{\alpha} \subset\{u \in$ $M(B):|\psi(u)|<1\}$. This contradicts [8, Theorem 3]. Thus $E_{x_{1}}=E_{x_{2}}=\cdots=$ $E_{x_{n}}=E_{x}$. As before we have that for $\bar{\psi} \in B_{m}, Z(\psi) \cap M(B) \subset E_{x}$, and $B_{m}=B[\bar{\psi}]$ if $Z(\psi) \cap M(B) \neq \emptyset$. This proves (ii).

Corollary 4.2. (i) Let $x \in M\left(H^{\infty}+C\right) \backslash M\left(L^{\infty}\right)$ and $B=H_{\text {supp } u_{x}}^{\infty}$. Then $B \subset$ $B_{m}$ if and only if $x$ is a minimal support point of $H^{\infty}[\bar{\psi}]$ for some interpolating Blaschke product $\psi$.

(ii) $B=B_{b}=B_{m}$ if and only if $x$ is not a minimal support point of $H^{\infty}[\bar{\psi}]$ for any interpolating Blaschke product.

Theorem 4.1(i) has also appeared in [12].

\section{Acknowledgements}

Research at MSRI is partially supported by NSF grant DMS-9022140. A major part of this paper was done while the author was at the Mathematical Sciences Research Institute. The author thanks the Institute for its support and also thanks Pamela Gorkin for many valuable discussions.

\section{References}

[1] S. Y. A. Chang, A characterization of Douglas subalgebras, Acta Math. 137 (1976), no. 2, 82-89. MR 55\#1074a. Zbl 332.46035.

[2] T. W. Gamelin, Uniform Algebras, Prentice-Hall, New Jersey, 1969. MR 53\#14137. Zbl 213.40401.

[3] P. Gorkin, K. Izuchi, and R. Mortini, Bourgain algebras of Douglas algebras, Canad. J. Math. 44 (1992), no. 4, 797-804. MR 94c:46104. Zbl 763.46046.

[4] P. Gorkin and R. Mortini, Interpolating Blaschke products and factorization in Douglas algebras, Michigan Math. J. 38 (1991), no. 1, 147-160. MR 92b:46083. Zbl 781.46037.

[5] C. J. Guillory and K. Izuchi, Maximal Douglas subalgebras and minimal support points, Proc. Amer. Math. Soc. 116 (1992), no. 2, 477-481. MR 92m:46075. Zbl 760.46046.

[6] Interpolating Blaschke products and nonanalytic sets, Complex Variables Theory Appl. 23 (1993), no. 3-4, 163-175. MR 95c:46076. Zbl 795.30031.

[7] Minimal envelopes of Douglas algebras and Bourgain algebras, Houston J. Math. 19 (1993), no. 2, 201-222. MR 94i:46067. Zbl 816.46048.

[8] H. Hedenmalm, Thin interpolating sequences and three algebras of bounded functions, Proc. Amer. Math. Soc. 99 (1987), no. 3, 489-495. MR 88c:46065. $\mathrm{Zbl} 617.46059$.

[9] K. Hoffman, Bounded analytic functions and Gleason parts, Ann. of Math. (2) 86 (1967), 74-111. MR 35\#5945. Zbl 192.48302. 
[10] K. Izuchi, QC-level sets and quotients of Douglas algebras, J. Funct. Anal. 65 (1986), no. 3, 293-308. MR 87f:46093. Zbl 579.46037.

[11] D. E. Marshall, Subalgebras of $L^{\infty}$ containing $H^{\infty}$, Acta Math. 137 (1976), no. 2, 91-98. MR 55\#1074b. Zbl 334.46061.

[12] R. Mortini and R. Younis, Douglas algebras which are invariant under the Bourgain map, Arch. Math. (Basel) 59 (1992), no. 4, 371-378. MR 94c:46105. Zbl 760.46050.

[13] R. Younis, Best approximation in certain Douglas algebras, Proc. Amer. Math. Soc. 80 (1980), no. 4, 639-642. MR 81j:46031. Zbl 444.30040.

Carroll Guillory: University of Louisiana at Lafayette, Lafayette, LA 70504, USA 


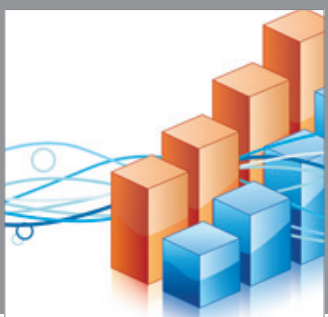

Advances in

Operations Research

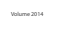

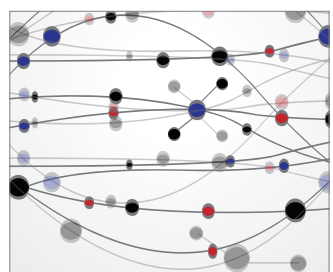

\section{The Scientific} World Journal
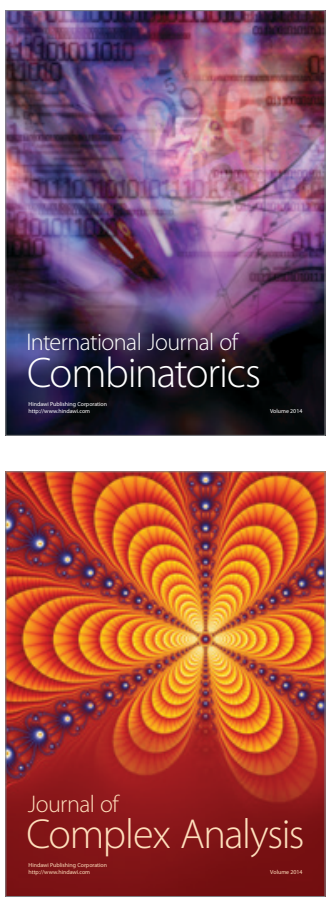

International Journal of

Mathematics and

Mathematical

Sciences
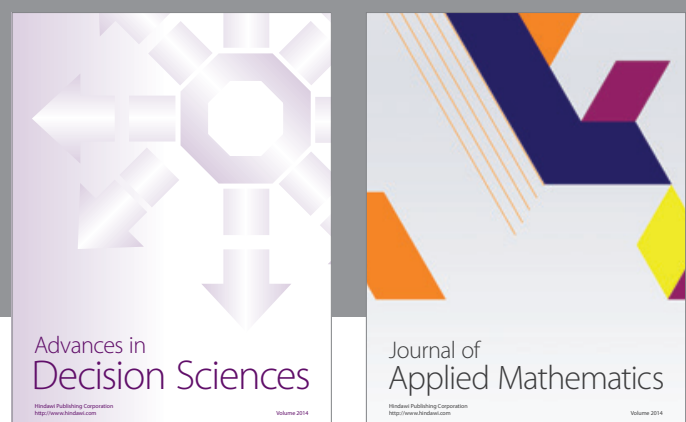

Journal of

Applied Mathematics
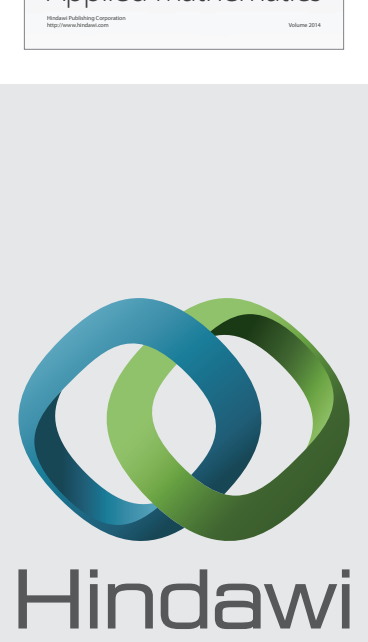

Submit your manuscripts at http://www.hindawi.com
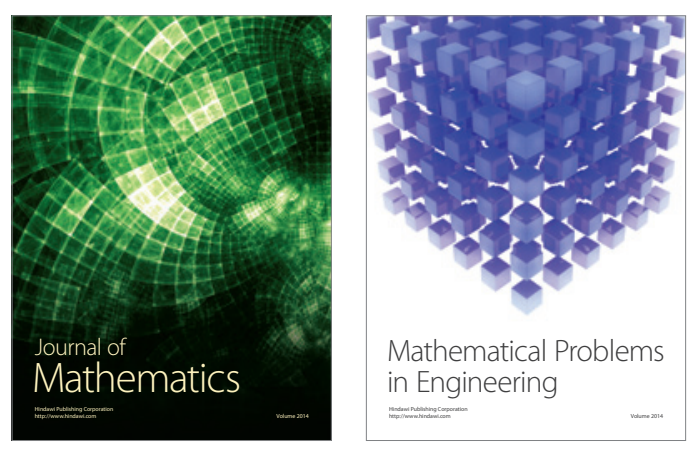

Mathematical Problems in Engineering
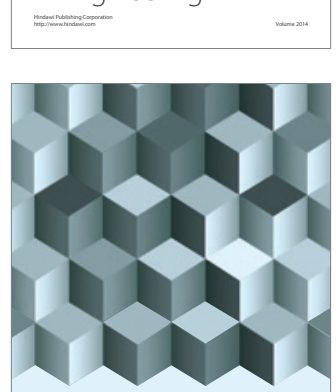

Journal of

Function Spaces
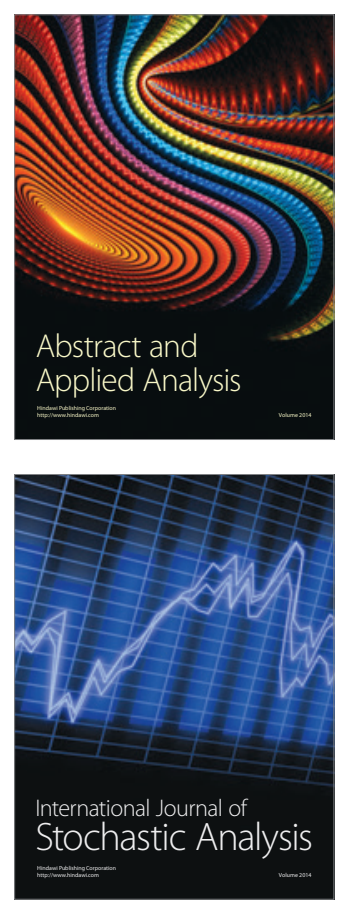

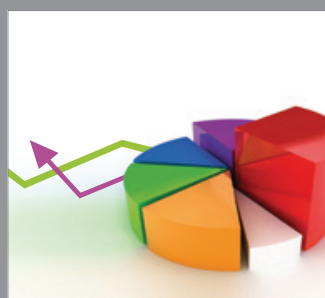

ournal of

Probability and Statistics

Promensencen
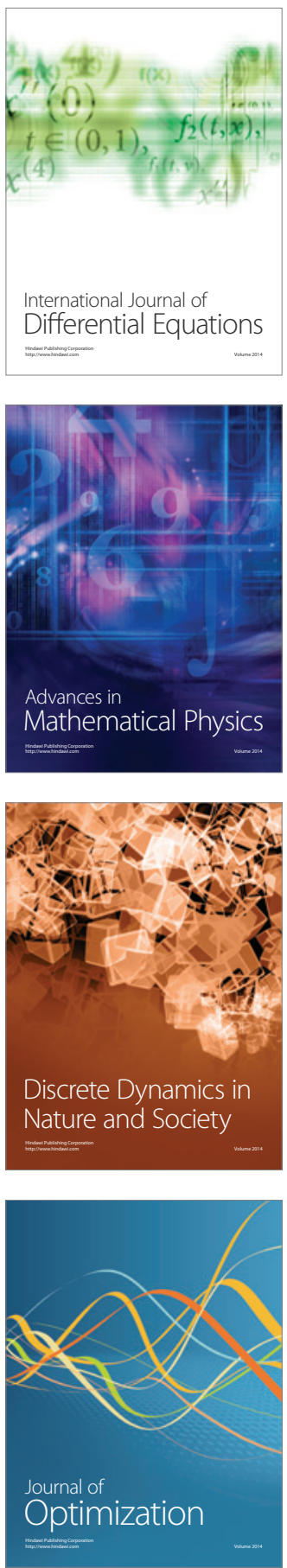